\title{
On the issue of effective protection of objects from vibration and shock
}

\author{
Viktor Nekhaev ${ }^{1}$, Viktor Nikolaev ${ }^{1, *}$, and Aleksandr Rauba ${ }^{1}$ \\ ${ }^{1}$ Omsk State Transport University, 644046, Marx av., 35, Omsk, Russia
}

\begin{abstract}
A brief review of the research on the solution of the problems of protecting objects from vibration and shock effects is performed. The ways to find the optimal values of vibration isolators are shown on the basis of the application of control theory, including with the use of feedforward control in systems of shockproof vibration isolation. The technique of designing quasi-invariant systems of vibration protection of objects is described and their high efficiency at various kinds of disturbing effect is shown, established as a result of numerical modeling and experimental researches.
\end{abstract}

\section{Introduction}

Modern high requirements to the safety of vehicles and the efficiency of their operation dictate the urgent need to improve the performance of their dynamic qualities, smooth running, reduce the vibration impact on their units, as well as on the human operator. Thus, when the railway rolling stock moves along the rail joints during the winter period, shock impacts (up to 40 -50 ton-force) on wheel sets and other units of the mechanical part occur, which significantly increases their stress-strain state, as well as the vibration level at the workplace of the locomotive crew. Large dynamic loads on the human operator also occur during the operation of tracked combat vehicles. In this regard, the task of ensuring effective vibration protection of objects, as well as ensuring their isolation from shock impact, has always been and remains extremely relevant.

\section{Existing methods for solving the problems of protection from vibration and shock}

The works of many well-known researchers are devoted to the solution of these problems. In the monograph [1], M.Z. Kolovsky describes the most common disturbing effects and classification of systems of vibration isolation of objects, describes methods of their mathematical analysis for various types of impacts. The problems of the dynamics of vibration protection systems are described in detail in the works of S.P. Timoshenko [2], Ya. G. Panovko [3], V.A. Svetlitsky [4], S. Crendall and A.A. Pervozvansky [5, 6], in which the dynamics of systems under deterministic and random impacts were investigated, and also during shock impact. The works $[7,8]$ are devoted to solving the problems of

\footnotetext{
* Corresponding author: NikolaevVA@omgups.ru
} 
vibration protection of machines and human operator, as well as modeling of oscillatory processes in "man-machine" systems. The limiting possibilities and optimization problems of elastic inertial vibration isolation systems were considered in [9].

Mathematical theory of optimal control developed by L.S. Pontryagin and his students [10] served as the basis for creating a new approach to studying and solving problems of vibration protection and shockproof vibration isolation of objects. One of its founders is M.Z. Kolovsky, [11], who used the methods of control theory to study the dynamics of mechanical oscillation systems. In this case, the vibration isolation system consisting of an elastic element (spring) and an oscillation damper can be considered as a control device U, forming a control force acting between the movable base and the protected object (Fig. 1).

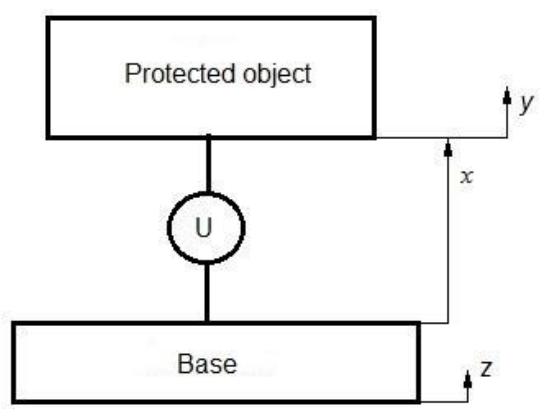

Fig. 1. Structural diagram of a mechanical oscillatory system containing a device for controlling the oscillations of a protected object

If we assume that the mass of the oscillating base is many times greater than the mass of the protected object, then the mathematical model describing the oscillations of the protected object under the kinematic disturbance has the form:

$$
m \ddot{x}+u=m \eta,
$$

where $x=y-z$-displacement of a protected object of mass $m$ relative to the oscillating base; $\mathrm{u}$ - control action on the protected object; $\eta=-\ddot{z}, \mathrm{z}-$ displacement of the base relative to the beginning of the absolute inertial frame of reference.

Scientific fundamentals and methods for calculating active and passive linear vibration protection systems are described in [12].

The work [13] is devoted to the search for optimal parameters of vibration protection systems. A number of problems on optimization of the characteristics and parameters of vibration protection devices for objects located on a vibrating base have been solved and described by N.N. Bolotnik in the monograph [14]. He set out a technique for determining the optimum values of the dampers of viscous and dry friction in various regions of the frequencies of the kinematic disturbance effect. By choosing the law of control of a shockproof insulator, the maximum displacement of the object relative to the base is minimized, provided that the magnitude of the interaction force per object does not exceed the specified value.

The theory and methods for optimal protection of objects from shock impact and vibration are described in detail in the monograph [15].

The urgency of the problem of choosing the optimal characteristics of shockproof isolators lies primarily in the fact that, as noted in [16], in cases where the period of elastic vibrations of the object is less than the duration of the shock impact, the use of shockproof vibration isolators leads to the opposite effect: the maximum value of the force acting on the object separated from the base by an isolation device does not decrease, but increases in 
comparison with the case of a rigid connection of the object with the base. Parametric synthesis defines a set of these devices that provide the ultimate capabilities of a shockproof isolator, and their main parameters are calculated. To this end, a class of vibration isolators consisting of a spring and a damper with power characteristics that form a control action of the following form are considered:

$$
u=c|x|^{n} \operatorname{sign} x+k|\dot{x}|^{r} \operatorname{sign} \dot{x}
$$

where $c$ and $k$ - the coefficients of spring stiffness and viscous friction of the damper, respectively, and exponents $n>0, k>0$, which can take different values. It is assumed that

$$
\text { when } t=t_{0}=0: x(0)=0, \dot{x}(0)=v_{0}
$$

and the shock impact occurs at the time $t=0$, which coincides with the moment when the control starts.

As a criterion for the quality of vibration isolation, the maximum displacement of the object relative to the base is adopted. It is established that one of the most acceptable shock isolators providing the ultimate capabilities of shockproof protection of an object is an isolator with a linear spring and quadratic damping:

$$
\mathrm{n}=1, \mathrm{r}=2, k=\frac{U}{v_{0}^{2}}, c=\frac{2 U^{2}}{m v_{0}^{2}},
$$

where $\mathrm{U}$ - a given positive constant limiting the magnitude of the control force to ensure the functional integrity of the object.

In the same work, the principle of feed-forward control is formulated, which in some cases significantly improves the quality of the shockproof protection of the object by preliminarily compressing the spring and releasing it at a certain time before the impact. The elastic element is pre-deformed and then released some time before the impact. The value of the preliminary deformation, the time of release of the isolator and the rigidity of the spring are chosen in the optimal way, depending on the intensity of the shock impact and the mass of the protected object. To implement this principle with the given mass of the protected object, it is required to predict the time of onset of the shock impact and its dynamic characteristics, such as, for example, impulse values and its duration. It is noted that for a model system with one degree of freedom, the use of a feed-forward spring isolator makes it possible to reduce the displacement of the protected object by 4 times in comparison with the limiting capabilities of the isolator of a non-feed-forward action, without increasing the maximum of the force module applied to the object from the side of the isolator.

This approach to the solution of the problem of parametric synthesis of a shockvibrating isolator containing elastic and dissipative elements was further developed in [17], which showed that the introduction of a dissipative element into the vibration isolator design allows improving the quality of protection by one and a half times in comparison with the optimal spring-based feed-forward isolator without damping.

At the same time, setting up such isolators for a feed-forward regime, as already noted, requires accurate determination of the impact time and its dynamic characteristics, which is not always possible, for example, when the actions are of a random nature. 


\section{The principle of invariance and its application for the creation of effective vibration and shock protection systems}

As practice shows, high modern requirements to the quality of vibration and shock protection cannot always be ensured by applying typical passive vibration isolation systems based on the use of elastic elements and vibration dampers, since these systems cannot always provide the desired indices of the dynamic qualities of the protected object in the low-frequency range of the disturbing effect and under shock impacts (type of transient process, stabilization of the object over a wide frequency range, etc.). One of the significant criteria, which in some cases influences the process of adopting new developments in vibration protection systems of objects, for example, when creating freight car trucks, are the strict requirements of a modern market economy that dictate the need to take into account the cost of a life cycle of their operation.

One of the effective ways to reduce the dynamic loading of objects under vibration and shock effects is the use of systems based on the application of the theory of invariance. The condition for the independence of one or several object coordinates from external disturbances is rigorously proved by Academician N.N. Luzin [18] and forms the basis of the theory of invariance.

As noted in [19], it can be briefly formulated as follows. If we consider a linear stationary system in a state space whose motion is described by a differential equation

$$
\dot{x}=A x+C w
$$

when $x_{0}=0$, and applying the Laplace transform, designate the vectors of state and disturbance by $\vec{x}(p)$ and $\vec{w}(p)$, respectively, we obtain

$$
\vec{x}(p)=(p E-A)^{-1} C \vec{w}(p) .
$$

In the scalar form, the last expression can be written as:

$$
x_{i}(p)=\frac{1}{\Delta} \sum_{\mu=1}^{n} \sum_{v=1}^{q} \alpha_{\mu i}(p) C_{\mu l} w_{v}(p),
$$

where $\alpha_{\mu i}(p)$-algebraic complement of the corresponding determinant element.

$$
\Delta=\left|\begin{array}{cccc}
p-a_{11} & -a_{12} & \cdots & -a_{1 n} \\
-a_{21} & p-a_{22} & \cdots & -a_{2 n} \\
\cdots & \cdots & \cdots & \cdots \\
-a_{n 1} & -a_{n 2} & \cdots & p-a_{n n}
\end{array}\right| .
$$

In order for the output $x_{i}$ to be invariant with respect to the disturbing effect $w_{v}$, it is necessary and sufficient that

$$
\frac{1}{\Delta} \sum_{\mu=1}^{n} \alpha_{\mu i}(p) C_{\mu l} \equiv 0
$$

If at least two coefficients are non-zero, for example, $C_{\mu v} \neq 0 . C_{\eta v} \neq 0$, and $C_{I v}=0$, than the equation 


$$
\sum_{\mu=1}^{n} \alpha_{\mu i}(p) C_{\mu l} \equiv 0
$$

can be satisfied identically, and condition (9) can be satisfied for finite values of the coefficients. This provision is a consequence of the two-channel principle formulated by Academician B.N. Petrov [20] and expresses the criterion of physical realizability of conditions (9). According to this principle, in order to achieve the invariance of the output coordinate of the system, it is necessary to have at least two channels of transmission of the impact between the point of application of the force and the point relative to the coordinate of which invariance is attained. Algebraic complements $\alpha_{\mu i}(p)$ when $\mu \neq i$ are polynomials of degree $n-2$ relative to $p$. Therefore, condition (10) is equivalent to the following equations:

$$
\sum_{\mu \neq i}\left[\frac{d^{\varepsilon}}{d p^{\varepsilon}} \alpha_{\mu i}(p)\right]_{\mu=0} C_{\mu \nu}=0, \varepsilon=0, \ldots, n-2 .
$$

The necessary structural condition reduces to the presence of two or more points of application of the disturbance $w_{v}$, and none of them should be in the channel with the output $x_{i}$ (Fig. 2, [19]).

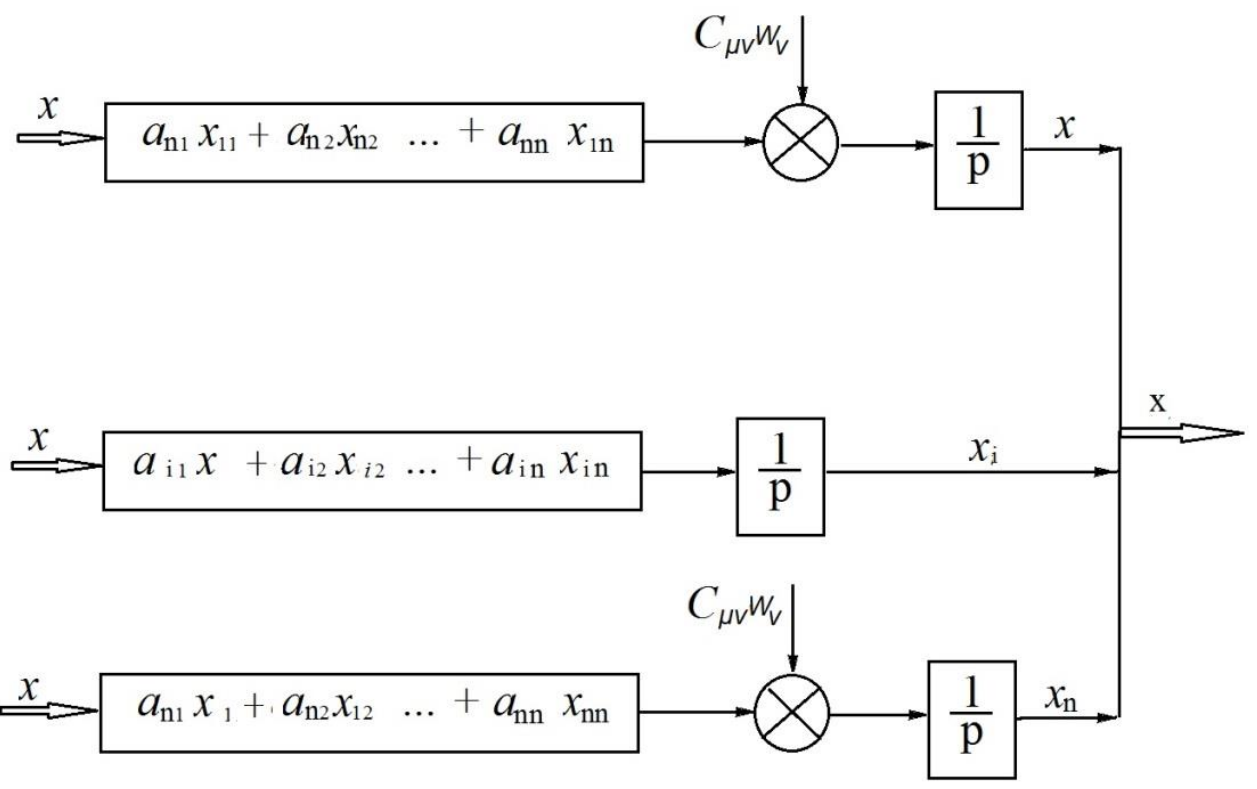

Fig. 2. Necessary condition for invariance at finite amplification factors is the presence of two-channels.

Then condition (9) becomes an identity $\alpha_{v i}(p) / \Delta \equiv 0$, which can only be satisfied if $\alpha_{v i}=\infty$, and the remaining coefficients are finite. Conditions (9) for given coefficients $C_{\mu \nu}$ can be regarded as equations with respect to parameters $a_{i k}$. For the case of the invariance of one output $x_{i}$ regarding to one disturbing effect $w_{v}$, as in our case, according 
to Fig. 1, the number of these equations is equal to $n-1$. The second sufficient sign of invariance is the provision of the requirement of physical realizability, i.e. satisfaction of the requirements of functional, dimensional, and strength constraints imposed on the values of the design parameters of the system. In this case, the second channel for transmitting the disturbing effect in practice can be realized in the form of an elastic element with two stable and one unstable equilibrium positions. In the latter, coinciding with the position of static equilibrium of the protected object, the potential energy of such a device has a maximum value. The behavior of such systems is described in [21]. A distinctive feature of vibration protection systems based on this method is that the compensating channel of the constructed quasi-invariant system of vibration protection of the object functions only under the action of the disturbance. It provides direct observance of the disturbing effect and forms a control action, i.e. a dynamic response directed towards a dynamic response of the main elastic element of the object's system of springs, as a result, in the dynamics, it is possible to provide high quality indices of vibration protection. At the same time, the nature of the disturbing effect is not of decisive importance, it is only necessary to know its modulus to form the necessary power characteristic of the compensating device for the given parameters of the basic elastic element.

\section{Design procedure of the compensating device}

When designing the compensating device for the axle box stage of suspension of the locomotive truck (Figure 3 ), if the damping is neglected, in the case of a kinematic disturbance due to locomotive motion along the track irregularities, the absolute invariance of the protected object is achieved by the condition [22]:

$$
S_{a} q+P_{V}(q)=0
$$

where $s_{a}, q$-the stiffness and deflection of the axle box stage of suspension $(q=y-z)$, respectively.

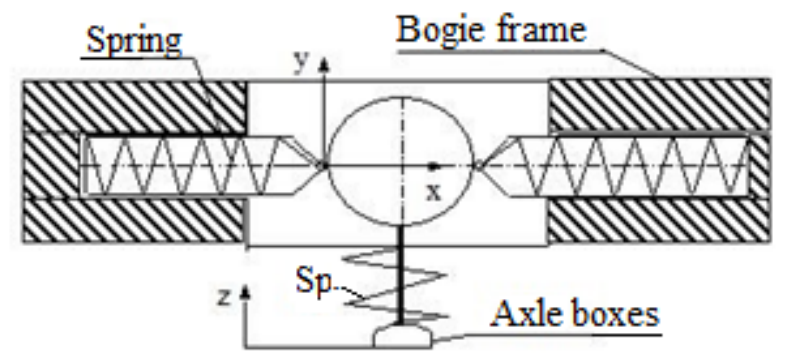

Fig. 3. Design scheme for spring suspension of the locomotive axle box stage with compensating device.

If the condition (12) is satisfied in the root mean square approximation, the optimization criterion can be written in the form

$$
J=\int_{-D}^{D}\left[Q(q)+P_{V}(q)\right]^{2} d q \rightarrow \min ,
$$

where $Q(q)=s_{a} q$ dynamic reaction of the axle box spring. $D$ - value of the desired protective movement of the vibration isolation device, determined by the deflection of the axle box stage of suspension. 
In the experimental devices of quasi-invariant vibration protection systems, a circle was used as a contact element, the shape of which is described by a curve of the second order

$$
-a_{11} x^{2}+a_{22} y^{2}+2 a_{1} x+2 a_{2} y=0
$$

whose coefficients have the following dimension $\quad a_{11}, a_{22}-\mathcal{M}^{2}, a_{1}, a_{2}-\mathcal{M}^{3}$.

Equation (14) for any $a_{11}, a_{22}, a_{1}, a_{2}$ should ensure conditions

$$
\left.x(y)\right|_{y=0}=0 ;\left.\quad \frac{d y}{d x}\right|_{x=0}=0,
$$

from where follows $a_{1}=0$.

As the mobile unit moves up or down from this position, the horizontal force decreases, which is determined by the expression

$$
P_{H}(x)=2 r_{c}[\Delta-y(x)]
$$

where $\Delta-$ initial deformation of additional elastic elements; $S_{c}-$ stiffness of an additional elastic element.

The power schemes that characterize the operation of the compensating device qualitatively are shown in Fig. 4. Let us consider the position of the system when it differs from the static one (Fig. $4 b, c)$.
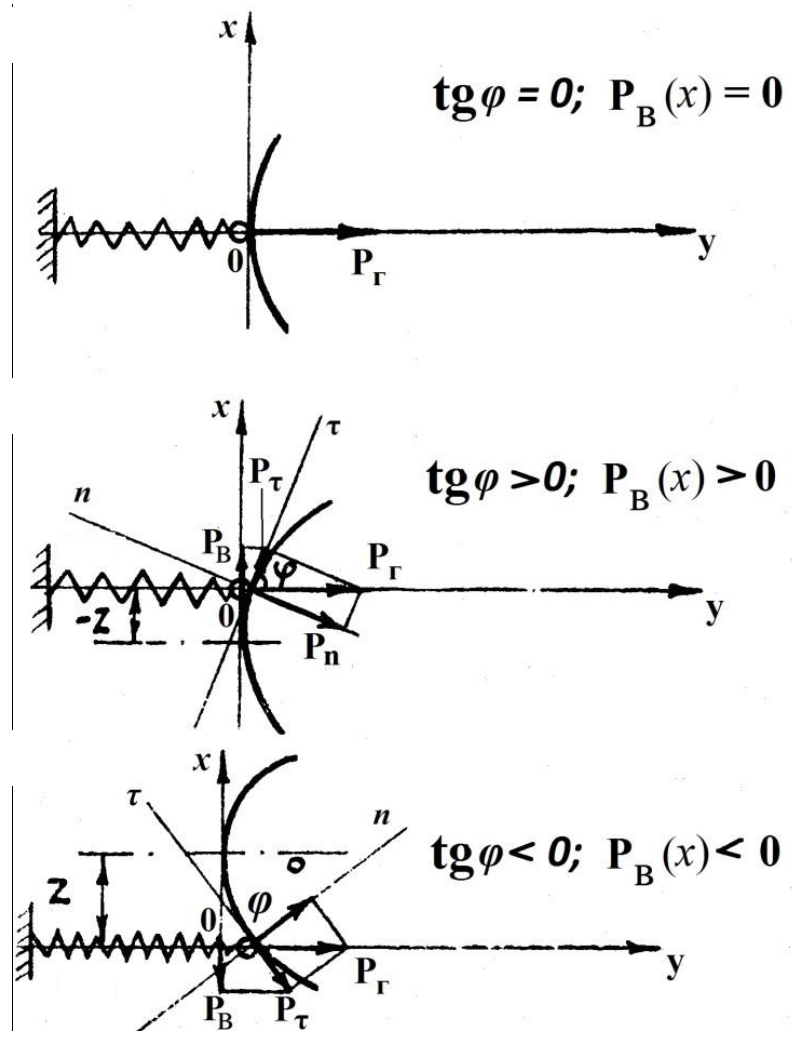

Fig. 4. Power schemes of the compensating device in its various positions. 
Decompose the horizontal power on the tangent and normal components:

$$
\left\{\begin{array}{l}
P_{\tau}=P_{H} \cos \varphi \\
P_{n}=P_{H} \sin \varphi
\end{array}\right.
$$

and project $P_{\tau}$ onto the axis $\mathrm{O} x$ :

$$
P_{V}(x)=P_{\tau}(x) \sin \varphi
$$

Substituting the expression $P_{\tau}$ from (17) into (18) and carrying out the necessary transformations, we obtain

$$
P_{V}(x)=\frac{1}{2} P_{H}(x) \sin 2 \varphi
$$

Considering that

$$
\begin{aligned}
& \sin 2 \varphi=\frac{2 \operatorname{tg} \varphi}{1+\operatorname{tg}^{2} \varphi} ; \\
& \operatorname{tg} \varphi=\frac{d x}{d t} ; \\
& \frac{d x}{d y}=-\frac{\sqrt{a_{2}^{2}+a_{11} a_{22} x^{2}}}{a_{11} x},
\end{aligned}
$$

we obtain the power characteristic of the device, expressed in terms of the coefficients of the curve (14)

$$
P_{\hat{A}}(x)=-2 a e_{\hat{e}}\left(\Delta-\frac{\sqrt{a_{2}^{2}+a_{11}^{2} x^{2}}-a_{2}}{a_{22}}\right) \times \frac{a_{11} x \sqrt{a_{2}^{2}+a_{11} a_{22} x^{2}}}{a_{2}^{2}+a_{11}\left(a_{11}+a_{22}\right) x^{2}} .
$$

The chosen values of the stiffness of the additional elastic element, the magnitude of its maximum potential energy in the static equilibrium position of the protected object, and the coefficients of the shape of the contact member allow realizing the power characteristic of the compensating device with odd symmetry

$$
P_{V}(q)=-\alpha x+\beta x^{3}
$$

ensuring the fulfillment of the condition (13).

\section{Results}

The values of the parameters of the compensating device that provide the imposed condition to the functional (13) are a zero approximation. After determining them, to ensure the stability of the dynamical system under consideration, their correction is performed. As a result, we obtain a quasi-invariant system with accuracy up to $\varepsilon$ with the corresponding power characteristic of the vibration isolator. Such a system of vibration protection of the railway vehicle does not require additional sources of energy to create regulators absorbing disturbances, the creation of which is discussed in $[23,24]$. The results of full-scale tests of 
the physical model of the human operator's vibration protection system are given in the table, from which it is evident that, in comparison with the input, the accelerations at the output decrease by $76.45 \mathrm{~dB}$.

Table 1. Dynamic indicators of the system under impact.

\begin{tabular}{|c|c|}
\hline Accelerations & Indicators \\
\hline$\ddot{Z}_{\text {in }}$ & $49,719 \mathrm{~g}(124,27 \mathrm{~dB})$ \\
\hline$\ddot{Z}_{\text {out }}$ & $0,00753 \mathrm{~g}(47,82 \mathrm{~dB})$ \\
\hline Efficiency & $76,45 \mathrm{~dB}$ \\
\hline
\end{tabular}

A comparative numerical study of mathematical models of the dynamics of electric locomotives 2ES6 with the random effect of the first variant with a typical scheme of the axle stage of suspension and the second variant with a compensating device, the scheme of which is shown in Fig. 3, in the speed range of $90-120 \mathrm{~km} / \mathrm{h}$ shows that the second variant provides a double reduction of vertical acceleration of the locomotive truck (Fig. 5).

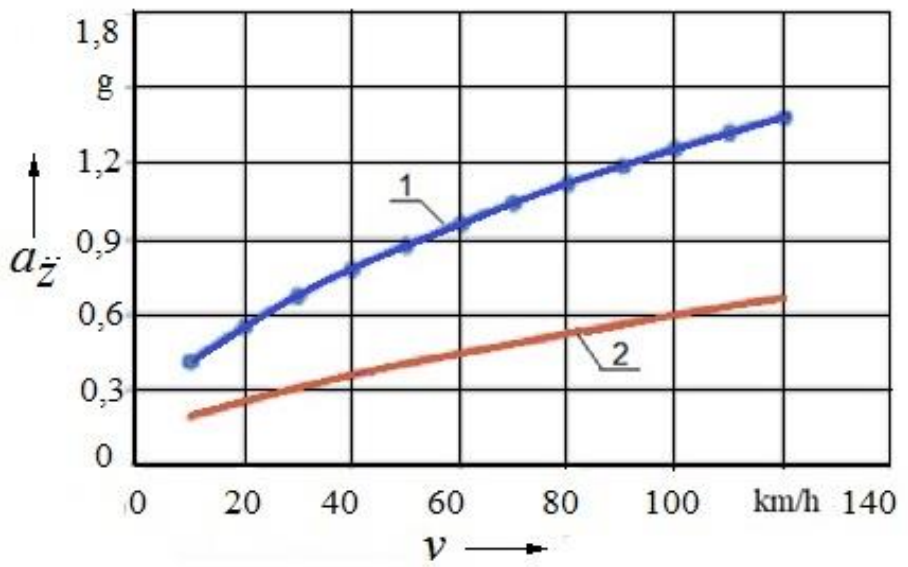

Fig. 5. Maximum acceleration of the locomotive truck: 1 - with a typical scheme of the axle stage of suspension; 2 - with compensating device.

\section{Conclusion}

The system of vibration isolation based on the principle of compensation of disturbances provides a high quality of protection of objects from kinematic disturbances and shock effects in comparison with typical schemes and with devices for feed-forward control under shock effects. This allows achieving high indicators of the dynamic qualities of the protected object with different character of the disturbing effect and increasing the efficiency of their operation.

\section{References}

1. M.Z. Kolovsky, Nonlinear theory of vibration protection systems (Nauka, Moscow, 1966)

2. S.P. Timoshenko, Oscillations in engineering (Science, Moscow, 1967)

3. Ya. G. Panovko, Fundamentals of applied theory of oscillations and shock (Mashinostroenie, Leningrad, 1976)

4. Random oscillations of mechanical systems (Mashinostroenie, Moscow, 1976) 
5. S.Y. Crendall, Numerical methods of analyses.-In Shock and vibration handbook (Mcgrow-Hill, N.Y., 1961)

6. S. Crendall and A.A. Pervozvansky, Random oscillations (Mir, Moscow, 1967)

7. K.V. Frolov, V.A. Furman, Applied theory of vibration protection systems (Mashinostroenie, Moscow, 1980)

8. L.A. Rybak, A.V. Sinev, A.I. Pashkov, Synthesis of active vibration isolation systems in space orbits (Janus-K, Moscow, 1997)

9. M.D. Genkin, V.M. Ryaboy, Elastic inertial vibration isolation systems. Limit capabilities, optimal structures (Nauka, Moscow, 1988)

10. L.S. Pontryagin, Mathematical theory of optimal processes (Nauka, Moscow, 1989)

11. M.Z. Kolovsky, Automatic control of vibration protection systems (Nauka, Moscow, 1976)

12. Dynamic properties of linear vibration protection systems (Nauka, Moscow, 1982)

13. R.I. Furunzhiev, Designing of optimal vibration protection systems (The Greatest School, Minsk, 1967)

14. N.N. Bolotnik, Optimization of vibration protection systems (Nauka, Moscow, 1983)

15. D.V. Balandin, N.N. Bolotnik. W.D. Pilkey, Optimal protection from impact, shock and vibration: theory and methods (Tailorand Francis Publishtrs, Philadelphia, 2001)

16. D.V. Balandin, N.N. Bolotnik, Mechanics of a solid body, 95 -109 (2003)

17. S.I. Purtsezov, Optimization of the shockproof protection of multi-mass elastic systems. Diss. cand. tech. sciences (N. Novgorod, 2006)

18. N.N. Luzin, Automation and Telemechanics 5, 4-66 (1940)

19. A.A. Krasovsky, A handbook on the theory of automatic control (Nauka, Moscow, 1987)

20. B.N. Petrov, Proceedings of the 1st All-Union Conference on the theory of invariance (Publishing House of the USSR Academy of Sciences, 1959)

21. Ya.G. Panovko, I.I. Gubanova, Stability and oscillations of elastic systems (Nauka, Moscow, 1967)

22. V.A. Nekchaev, V.A.Nikolaev, Synthesis of invariant vibration protection system (theory and practice) Nonlinear vibration problems (DWH-Polish Scientific Publishers, Warszawa, 1993)

23. T.A. Tibilov, T. Tsisovski, Thes. rep. IV scientific and practical conference Resourcesaving technologies in railway transport (MIIT, Moscow, 2001)

24. T. Tsisovski, Vestnik MIITa 6, 20-25 (2001) 